Supplement of Biogeosciences, 13, 781-794, 2016

http://www.biogeosciences.net/13/781/2016/

doi:10.5194/bg-13-781-2016-supplement

(C) Author(s) 2016. CC Attribution 3.0 License.

(c) (i)

Supplement of

\title{
Changing nutrient stoichiometry affects phytoplankton production, DOP accumulation and dinitrogen fixation - a mesocosm experiment in the eastern tropical North Atlantic
}

\section{J. Meyer et al.}

Correspondence to: J. Meyer (jumeyer@geomar.de)

The copyright of individual parts of the supplement might differ from the CC-BY 3.0 licence. 
Supplemental Information to 'Changing nutrient stoichiometry affects phytoplankton production, DOP build up and dinitrogen fixation - a mesocosm experiment in the eastern tropical North

Atlantic‘

2 Table S1: Nominal and measured nutrient concentrations after the addition of nitrate or phosphate to the 3 mesocosms in both experimental runs.

\begin{tabular}{|c|c|c|c|c|c|c|c|c|c|}
\hline Run & Treat ID & $\begin{array}{l}\mathrm{NO}_{3}{ }^{-} \mathrm{nom} \\
{\left[\mu \mathrm{mol} \mathrm{L}^{-1}\right]}\end{array}$ & $\begin{array}{l}\mathrm{PO}_{4}{ }^{3-} \text { nom } \\
{\left[\mu \mathrm{mol} \mathrm{L}^{-1}\right]}\end{array}$ & $\begin{array}{l}\mathrm{SiO}_{2} \text { nom } \\
{\left[\mu \mathrm{mol} \mathrm{L}^{-1}\right]}\end{array}$ & $\mathbf{N}: \mathbf{P}$ nom & $\begin{array}{c}\mathrm{NO}_{3}{ }^{-} \\
{[\mu \mathrm{mol} \mathrm{L-1]}}\end{array}$ & $\begin{array}{c}\mathrm{PO}_{4}{ }^{3-} \\
{\left[\mu \mathrm{mol} \mathrm{L}^{-1}\right]}\end{array}$ & $\begin{array}{c}\mathrm{SiO}_{2} \\
{\left[\mu \mathrm{mol} \mathrm{L}^{-1}\right]}\end{array}$ & $\mathbf{N}: \mathbf{P}$ \\
\hline$\overline{11}$ & $12.0 \mathrm{~N} / 0.75 \mathrm{P}$ & 12 & 0.75 & 15 & 16 & 11.52 & 0.73 & 15.22 & 15.78 \\
\hline 1 & $12.0 \mathrm{~N} / 0.75 \mathrm{P}$ & 12 & 0.75 & 15 & 16 & 10.97 & 0.68 & 14.97 & 16.11 \\
\hline 1 & $12.0 \mathrm{~N} / 0.75 \mathrm{P}$ & 12 & 0.75 & 15 & 16 & 10.63 & 0.52 & 15.04 & 20.47 \\
\hline 1 & $6.35 \mathrm{~N} / 1.10 \mathrm{P}$ & 6.35 & 1.1 & 15 & 5.76 & 5.66 & 1.00 & 15.06 & 5.66 \\
\hline 1 & $12.0 \mathrm{~N} / 1.25 \mathrm{P}$ & 12 & 1.25 & 15 & 9.6 & 10.74 & 1.14 & 15.01 & 9.39 \\
\hline 1 & $12.0 \mathrm{~N} / 1.25 \mathrm{P}$ & 12 & 1.25 & 15 & 9.6 & 11.16 & 1.12 & 15.33 & 9.95 \\
\hline 1 & $12.0 \mathrm{~N} / 1.25 \mathrm{P}$ & 12 & 1.25 & 15 & 9.6 & 10.89 & 1.09 & 15.13 & 9.97 \\
\hline 1 & $12.0 \mathrm{~N} / 1.75 \mathrm{P}$ & 12 & 1.75 & 15 & 6.86 & 10.55 & 1.57 & 14.97 & 6.74 \\
\hline 1 & $12.0 \mathrm{~N} / 0.75 \mathrm{P}$ & 12 & 0.75 & 15 & 16 & 10.82 & 0.61 & 15.10 & 17.64 \\
\hline 1 & $12.0 \mathrm{~N} / 1.75 \mathrm{P}$ & 12 & 1.75 & 15 & 6.86 & 10.82 & 1.58 & 14.90 & 6.86 \\
\hline 1 & $12.0 \mathrm{~N} / 1.75 \mathrm{P}$ & 12 & 1.75 & 15 & 6.86 & 11.07 & 1.53 & 15.01 & 7.24 \\
\hline 1 & $12.0 \mathrm{~N} / 0.25 \mathrm{P}$ & 12 & 0.25 & 15 & 48 & 11.16 & 0.15 & 15.12 & 76.78 \\
\hline 1 & $12.0 \mathrm{~N} / 0.25 \mathrm{P}$ & 12 & 0.25 & 15 & 48 & 11.18 & 0.16 & 15.00 & 69.80 \\
\hline 1 & $17.65 \mathrm{~N} / 1.10 \mathrm{P}$ & 17.65 & 1.1 & 15 & 16 & 16.90 & 1.01 & 15.27 & 16.75 \\
\hline 1 & $12.0 \mathrm{~N} / 0.25 \mathrm{P}$ & 12 & 0.25 & 15 & 48 & 11.33 & 0.15 & 15.15 & 75.77 \\
\hline 2 & $12.0 \mathrm{~N} / 0.75 \mathrm{P}$ & 12 & 0.75 & 15 & 16 & 12.58 & 0.47 & 14.51 & 27.00 \\
\hline 2 & $12.0 \mathrm{~N} / 0.75 \mathrm{P}$ & 12 & 0.75 & 15 & 16 & 12.36 & 0.51 & 14.18 & 24.32 \\
\hline 2 & $12.0 \mathrm{~N} / 0.75 \mathrm{P}$ & 12 & 0.75 & 15 & 16 & 12.61 & 0.51 & 14.34 & 24.72 \\
\hline 2 & $6.35 \mathrm{~N} / 0.40 \mathrm{P}$ & 6.35 & 0.4 & 15 & 15.99 & 6.91 & 0.18 & 14.63 & 39.35 \\
\hline 2 & $17.65 \mathrm{~N} / 1.10 \mathrm{P}$ & 17.65 & 1.1 & 15 & 16.05 & 18.43 & 0.79 & 14.47 & 23.45 \\
\hline 2 & $20.0 \mathrm{~N} / 0.75 \mathrm{P}$ & 20 & 0.75 & 15 & 26.67 & 20.57 & 0.47 & 15.09 & 43.92 \\
\hline 2 & $20.0 \mathrm{~N} / 0.75 \mathrm{P}$ & 20 & 0.75 & 15 & 26.67 & 20.60 & 0.45 & 14.16 & 45.92 \\
\hline 2 & $20.0 \mathrm{~N} / 0.75 \mathrm{P}$ & 20 & 0.75 & 15 & 26.67 & 21.90 & 0.45 & 15.18 & 48.81 \\
\hline 2 & $4.00 \mathrm{~N} / 0.75 \mathrm{P}$ & 4 & 0.75 & 15 & 5.33 & 4.62 & 0.45 & 15.33 & 10.38 \\
\hline 2 & $17.65 \mathrm{~N} / 0.40 \mathrm{P}$ & 17.65 & 0.4 & 15 & 44.46 & 18.47 & 0.22 & 15.36 & 84.31 \\
\hline 2 & $4.00 \mathrm{~N} / 0.75 \mathrm{P}$ & 4 & 0.75 & 15 & 5.33 & 4.49 & 0.47 & 14.92 & 9.59 \\
\hline 2 & $4.00 \mathrm{~N} / 0.75 \mathrm{P}$ & 4 & 0.75 & 15 & 5.33 & 3.99 & 0.49 & 15.68 & 8.17 \\
\hline 2 & $2.00 \mathrm{~N} / 0.75 \mathrm{P}$ & 2 & 0.75 & 15 & 2.67 & 2.06 & 0.46 & 16.39 & 4.52 \\
\hline 2 & $6.00 \mathrm{~N} / 1.03 \mathrm{P}$ & 6.00 & 1.03 & 15 & 5.77 & 6.69 & 0.78 & 15.46 & 8.55 \\
\hline 2 & $2.00 \mathrm{~N} / 0.75 \mathrm{P}$ & 2 & 0.75 & 15 & 2.67 & 1.87 & 0.56 & 17.64 & 3.33 \\
\hline 2 & $2.00 \mathrm{~N} / 0.75 \mathrm{P}$ & 2 & 0.75 & 15 & 2.67 & 2.71 & 0.48 & 15.04 & 5.60 \\
\hline
\end{tabular}


8 Table S2: Mass balance. $\mathrm{P}$ pool $=\mathrm{PO}_{4}{ }^{3-}+\mathrm{DOP}+\mathrm{POP}$. $\mathrm{P}$ pool $\mathrm{x}=$ undetermined $\mathrm{P}$ pool, which represents 9 a combination of measurement errors and wall growth. Statistical significance (t-test) between pool sizes 10 on day 2 and day 8 is denoted by asterisks. No t-tests were conducted when only one replicate was 11 available.

\begin{tabular}{|c|c|c|c|c|c|c|}
\hline Run & Treat ID & Replicates & $\begin{array}{c}\text { P pool day } 2 \\
{\left[\mu \mathrm{mol} \mathrm{L}{ }^{-1}\right]}\end{array}$ & $\begin{array}{c}\text { P pool day } 8 \\
{\left[\mu \mathrm{mol} \mathrm{L} L^{-1}\right]}\end{array}$ & $\begin{array}{c}\text { P poolx } \\
{[\mu \mathrm{mol} \mathrm{L}-1]}\end{array}$ & $\begin{array}{c}\text { P poolx } \\
{[\%]}\end{array}$ \\
\hline 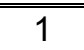 & 6.35N/1.10P & $\overline{11}$ & 1.52 & 1.12 & -0.40 & 26 \\
\hline 1 & $12.0 \mathrm{~N} / 0.25 \mathrm{P}$ & 3 & $0.53 \pm 0.07$ & $0.45 \pm 0.03$ & -0.08 & 15 \\
\hline 1 & $12.0 \mathrm{~N} / 0.75 \mathrm{P}$ & 4 & $0.97 \pm 0.12$ & $0.71 \pm 0.11$ & -0.26 & 27 \\
\hline 1 & $12.0 \mathrm{~N} / 1.25 \mathrm{P}$ & 3 & $1.42 \pm 0.06$ & $1.02 \pm 0.10$ & $-0.40^{*}$ & 28 \\
\hline 1 & $12.0 \mathrm{~N} / 1.75 \mathrm{P}$ & 3 & $1.86 \pm 0.05$ & $1.42 \pm 0.20$ & $-0.44^{*}$ & 24 \\
\hline 1 & $17.65 \mathrm{~N} / 1.10 \mathrm{P}$ & 1 & 1.34 & 0.92 & -0.42 & 31 \\
\hline 2 & $2.00 \mathrm{~N} / 0.75 \mathrm{P}$ & 3 & $0.88 \pm 0.11$ & $0.84 \pm 0.19$ & -0.04 & 5 \\
\hline 2 & $4.00 N / 0.75 \mathrm{P}$ & 3 & $0.89 \pm 0.06$ & $0.75 \pm 0.12$ & -0.14 & 16 \\
\hline 2 & $6.00 \mathrm{~N} / 1.03 \mathrm{P}$ & 1 & 1.13 & 0.71 & -0.42 & 37 \\
\hline 2 & $6.35 \mathrm{~N} / 0.40 \mathrm{P}$ & 1 & 0.56 & 0.54 & -0.02 & 4 \\
\hline 2 & $12.0 \mathrm{~N} / 0.75 \mathrm{P}$ & 3 & $0.89 \pm 0.07$ & $0.76 \pm 0.10$ & -0.13 & 15 \\
\hline 2 & $17.65 / 0.40 \mathrm{P}$ & 1 & 0.56 & 0.48 & -0.08 & 14 \\
\hline 2 & $17.65 \mathrm{~N} / 1.10 \mathrm{P}$ & 1 & 1.19 & 1.15 & -0.04 & 3 \\
\hline 2 & $20.0 \mathrm{~N} / 0.75 \mathrm{P}$ & 3 & $0.88 \pm 0.03$ & $0.73 \pm 0.10$ & -0.15 & 17 \\
\hline
\end{tabular}

